cycle was conducted after introducing the Cirrhosis care bundle.

Results

\begin{tabular}{lll} 
Astracts PTH-110 Table 1 & \multicolumn{2}{c}{ Compliance with quality standards } \\
before and after introduction of Cirrhosis Care Bundle
\end{tabular}

${ }^{*} \mathrm{SBP}=$ spontaneous bacterial peritonitis

Conclusions After formally adopting the Cirrhosis care bundle, we have seen an improvement in the frequency of ascitic taps performed, treatment of spontaneous bacterial peritonitis, prescription of VTE prophylaxis and hepatic encephalopathy. Our targets have certainly approved but not enough to meet quality standards. It was decided to conduct third cycle to improve our targets. We decided to educate doctors in A and E because patients spend more time there before being transferred to MAU due to pressures on NHS. The teaching involved identifying patients presenting with signs and symptoms of decompensated liver disease and education about the cirrhosis care bundle, its importance. Third cycle is in progress and showing promising Results.

\section{PTH-111 IMPROVING END OF LIFE CARE FOR PATIENTS WITH LIVER DISEASE}

${ }^{1}$ Sam Thomson*, ${ }^{2}$ Kim Batchelor, ${ }^{1}$ Stephen Kriese, ${ }^{2}$ Suzanne Ford-Dunn. ${ }^{1}$ Western Sussex Hospitals NHS Foundation Trust, Worthing, UK; ${ }^{2}$ St Barnabas House Hospice, Worthing, UK

10.1136/gutjnl-2018-BSGAbstracts.267
Introduction Liver disease is the 5th commonest cause of death in the UK and the only major cause of death still increasing. National data show that $73 \%$ of deaths occur in hospital and few patients are referred to palliative care services (PC) despite complex symptoms and psychosocial needs. Although preferred place of death for patients with end stage liver disease (ESLD) is unknown, studies of other diagnoses show the majority of patients prefer to die at home, rather than hospital. We aimed to increase access to PC for patients with ESLD and to ascertain preferred place of death.

Methods We commenced a monthly ESLD MDT, comprising Hepatology and PC Consultants, community ESLD Clinical Nurse Specialist (CNS), Alcohol CNS, Social Worker and hospital PC CNS. Patients were identified as outpatients and inpatients and referred to the MDT for discussion. The MDT reviewed patient needs, coordinated care and initiated referrals to additional community services and ESLD CNS. Patients referred to the ESLD CNS received holistic assessment with advanced care planning and contingency plans for future acute decompensation events.

Results In the first 12 months of the new MDT there were 43 deaths with ESLD in our locality, 60\% in hospital, 37\% in community (home/hospice); contrasting to $73 \%$ and $26 \%$ nationally. $79 \%$ of all patients were known to PC at the time of death. Of 22 patients that expressed a preference for place of death, 11 chose home and 11 hospice; none preferred to die in hospital. Of 22 patients under the ESLD CNS in the community, $73 \%$ died out of hospital (7 home, 9 hospice). $68 \%$ of patients under the ESLD CNS died in their preferred place of care. Of 26 who died in hospital 30\% died on the acute medical unit, HDU or ITU, $30 \%$ on the Gastroenterology ward and the remainder (40\%) on outlying wards.

Conclusions Most patients with ESLD prefer to die out of hospital, consistent with other terminal illnesses. Although ESLD patients present a challenging symptom burden it appears that an MDT approach including a dedicated CNS can help increase referrals to PC, and help more patients die in their preferred place. However, a third of those dying in hospital did so in an acute environment or while receiving intensive care and therefore were unlikely to have received adequate PC. A notable number also died on non-specialist outlying wards which may imply they too did not receive wholly integrated specialist or PC during their terminal phase.

Although the preliminary Results of our local intervention are promising, we recognise that more can still be done and encourage a continued focus on improving end of life support and care planning for patients with ESLD.

\section{PTH-112 INVESTIGATION UPDATE: A CLUSTER OF GENOTYPE A2 'PRISONER VARIANT' ACUTE HEPATITIS B INFECTION}

${ }^{1,2}$ Sebastian Wallace*, ${ }^{2}$ Nicholas Young, ${ }^{2}$ Samreen ljaz, ${ }^{2}$ Sema Mandal. ${ }^{1}$ Royal Cornwall Hospital, Truro, UK; ${ }^{2}$ Public Health England, London, UK

\subsection{6/gutjnl-2018-BSGAbstracts.268}

Introduction Between April 2012 and May 2015, a cluster of acute Hepatitis B virus (HBV) infection was investigated by Public Health England (PHE) in Southwest England. Compared nationally, it was observed that cases were above the average age, all male and did not belong to typical 'highrisk' groups; with a majority reporting no risk-factors for $\mathrm{HBV}$ exposure. It was hypothesised that these cases were 
linked by a common source of transmission or a shared behaviour and that this link could be demonstrated by phylogenetic analysis.

Methods A case definition of men, aged 35-75, presenting with acute HBV, with no recorded risk factors, was adopted. The PHE Database 'HP zone' was used to compile a line list of new acute HBV matching the case definition within the undisclosed county, from January 2012 to January 2015. Inclusion criteria for all cases required HBV core IgM positive status with clinical symptoms or biochemical markers consistent with acute hepatitis. Data on risk factors was compiled, as part of routine investigation, from the PHE acute HBV questionnaire using open and focussed questioning. Serum specimens on the line list were sent to the PHE laboratory, Colindale for phylogenetic analysis.

Results 33 cases of acute HBV were identified with 19 men fitting the case definition. The line list was expanded to include the remaining 7 men aged $35-75$ in the same region and timeframe who did report risk factors for HBV exposure (total=26). 6 cases did not have stored serum for genotyping. 17 cases of HBV Genotype A2 were identified, 14 met the exact case definition with no risk factors with the remaining 3 reporting high-risk sexual activity with men. 3 Cases of Genotype D were identified, all of which reported risk factors. 14 of the 19 cases that fitted the definition described themselves as heterosexual and married at the time of diagnosis. All spouses tested negative for HBV.

Of the 17 A2 genotypes 16 were 99\%-100\% identical and 12 were $99.7 \%-100 \%$ identical at the nucleotide level with one case differing by a single base pair at the HBsAg region. All cases were of a single, stable strain known as the 'Prisoner Variant', which has increased in prevalence from 1990, primarily in the prison population of north England.

Conclusions The genotypic link of at least 16 cases with a non-prevalent strain of $\mathrm{HBV}$ indicates a common source of infection. The lack of confirmed risk factors in so many cases may indicate a shift in the 'epi-picture' with new behaviours making at-risk groups harder to identify for targeted healtheducation or vaccination. The cluster remains active with further cases being investigated.

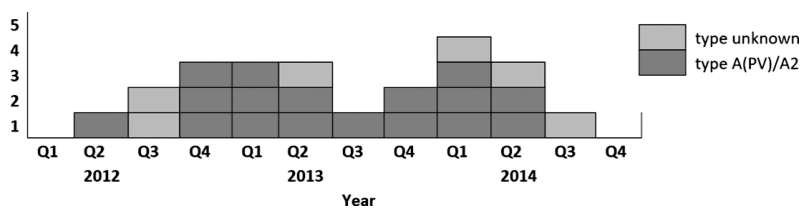

Abstract PTH-112 Figure 1 Epidemic curve displaying cases of acute Hep B per quarter 2012 to 2015; cases other than A/A2 excluded

\section{PTH-113 THE SUCCESS OF KAFFES STENT INSERTIONS FOR POST LIVER TRANSPLANT ANASTOMOTIC STRICTURES}

Deepak Joshi* ${ }^{*}$ Ben Warner*, Phillip Harrison, John Devlin, David Reffitt, Yasser El-Sherif, Shirin Khorsandi, Andreas Prachalias, Miriam Cortes Cerisuelo, Krishna Menon, Wayel Jassem, Parthi Srinivasan, Hector Vilca-Melendez, Michael Heneghan, Nigel Heaton. Institute of Liver Studies, King's College Hospital, London, UK

\subsection{6/gutjnl-2018-BSGAbstracts.269}

Introduction Biliary anastomotic strictures (AS) occur in around $30 \%$ of patients following liver transplantation and are treated by endoscopic dilatation and plastic stent (PS) insertion. However, AS frequently recur and patients require multiple procedures. The Kaffes stent (KS, Taewoong Medical) is a removable, covered metal stent designed to be deployed across AS.

Methods To examine outcomes in patients with AS, we compared a recent cohort of patients treated using KS with a historical cohort of patients who received PS.

Results The 22 patients (12 females) treated by KS had mean age 55 (range 22-69) years; 11 patients had DBD and 11 DCD grafts; mean cold ischaemia time was 9.6 \pm 3.3 hours. Four patients had failed previous treatment with PS. To date, 16 patients have had KS removed. The 69 patients $(20$ female) treated by PS were similar, mean age 51 (range 2879) years; 47 patients had DBD and 22 DCD grafts; mean cold ischaemia time was $8.9 \pm 3.1$ hours.

AS resolved after one deployment of KS in 14 out of 16 patients (88\%) compared to 26 out of 69 patients (38\%) receiving their first PS (Relative Risk of persistent stricture (KS vs PS $)=0.2$, 95\% CI $0.05-0.74 ; \mathrm{p}=0.016$; number to treat by KS for one benefit $=2$, 95\% CI 1.3-4.0). There were no complications, including stent migration, after KS compared to $6(8.4 \%)$ in the PS group (3 cholangitis, 2 pancreatitis, and 1 bleeding). All KS were removed successfully, although 1 stent needed 2 attempts because of wire migration. Following initial ERCP, PS patients required more ERCPs (mean 2.71 vs 1.13 more; $\mathrm{p}<0.01$ ) and $32 \%$ required biliary reconstruction.

Conclusion Our data indicate that the $\mathrm{KS}$ is a promising Method for managing post-transplant AS because the majority of strictures are treated by deployment of a single stent at first ERCP.

\section{PTH-114 REAL-WORLD PERFORMANCE OF NON-INVASIVE IMAGING MODALITIES IN THE DETECTION OF COMMON BILE DUCT STONES (CBDS)}

Javed Iqbal* ${ }^{*}$ Benjamin Giles, Chris Palmer-Jones, William Crinnion, Mohammed Syed, Simon Phillpotts, Sudeep Tanwar. Whipps Cross University Hospital, Barts Health NHS Trust, London, UK

\subsection{6/gutjnl-2018-BSGAbstracts. 270}

Introduction Cholangiography at endoscopic retrograde cholangio-pancreatography (ERCP), the reference standard for detecting CBDS, is not practicable as the primary Method for identifying CBDS as it is invasive and has an associated morbidity. With regard to the non-invasive identification of CBDS, the current BGS guidance outlines pooled sensitivities of $73 \%$, $69 \%-87 \%$ and $93 \%$ for ultrasound (US), computed tomography (CT) and magnetic resonance cholangio-pancreatography (MRCP) respectively. In this study we report the real-world sensitivity of these imaging modalities to detect CBDS.

Method All cases of CBDS confirmed at ERCP over 12 months were identified prospectively. The imaging modalities employed prior to ERCP were identified and evaluated for the presence or absence of duct dilatation $(>6 \mathrm{~mm})$ or intraductal contents (stone or sludge) thereby defining the sensitivity of these parameters for detecting CBDS. 95\% confidence interval (CI) of the sensitivities was calculated.

Results In total, 102 patients had ERCP for CBDS (female $57 \%)$ after presenting with pain $(83 \%)$ and jaundice $(45 \%$. Liver function tests were abnormal in $82 \%$. 48 patients had CBDS $\geq 1 \mathrm{~cm}$ (maximum size $35 \mathrm{~mm}$, mean $9.6 \mathrm{~mm}$ ). All US scans in this study were performed by sonographers. 